\title{
Development of a Novel Tablet Machine for a Tiny Amount of Powder and Evaluation of Capping Tendency
}

\author{
Hideya Nakamura, * Yui Sugino, Tomohiro Iwasaki, and Satoru Watano \\ Department of Chemical Engineering, Osaka Prefecture University; 1-1 Gakuen-cho, Naka-ku, Sakai, Osaka 599-8531, \\ Japan. Received August 12, 2011; accepted September 27, 2011; published online September 28, 2011
}

\begin{abstract}
A novel single punch tablet machine was developed for a tiny amount of powder sample. This tablet machine mainly consists of upper and lower punches, single die, and conical powder feeder equipped with micro-vibrators. By using the powder feeder, mass of discharged powder can be maintained constant even if a tiny amount of powder having poor flowability is used. Motions of both upper and lower punches can be set arbitrarily. Thus, this machine enables us to prepare tablets with a tiny amount of powder sample under the same compression mechanism as conventional rotary tablet machines. Performance of the developed tablet machine was evaluated in a continuous direct tableting using a model powder with poor flowability. Thirty-four tablets $(195 \mathrm{mg} \times 34)$ having acceptable properties can be successfully prepared using no more than $\mathbf{1 0 . 0} \mathrm{g}$ of a powder sample. We then proposed a novel in-die evaluation method of capping tendency. A new phase diagram consisting of the elastic recovery energy and the plastic deformation energy was proposed. These energies were calculated from a force-displacement profile, continuously monitored by the developed tablet machine. The results indicate that by using the new diagram the capping tendency of tablets prepared from various model powders can be well discriminated. The developed tablet machine and proposed evaluation method can contribute to a significant cost reduction and speeding up of formulation studies of oral dosage form.
\end{abstract}

Key words tableting; tiny amount; capping; force-displacement profile

Tablet is the most common pharmaceutical oral dosage form due to the patient's acceptability, high drug stability, and high productivity. ${ }^{1}$ A formulation study of a tableting process is very important for stable manufacturing of products having desired properties. Design space of a tableting process basically consists of two components: (1) material attributes of powders such as elasticity and plasticity, and (2) operating parameters in a manufacturing process such as compaction load. This means that various kinds of pharmaceutical powders should be tested under various compositions as well as various operating parameters. However, in an early stage of formulation study, new active pharmaceutical ingredients (APIs) are generally very expensive and its available quantity is very tiny, ${ }^{2,3)}$ This strictly restricts a number of experiments and makes it difficult to sufficiently investigate the design space of a tableting process. Therefore, if tablets can be successfully prepared from a tiny amount of powder, it is possible to efficiently investigate its design space, resulting in significant cost reduction and speeding up of a new drug development.

Rotary tablet machines are widely used for production of pharmaceutical tablets. In rotary tablet machines, both lower and upper punches compress a powder and tablets can be continuously prepared by a two-stage compaction, in which a powder is compressed twice. ${ }^{4)}$ Most of the rotary tablet machines available on the market have been developed as a pilot-scale machine or manufacturing-scale machine. Thus, to prepare tablets using rotary tablet machines, a large amount of powders (a few hundred grams at least) is needed. Single punch tablet machines are also common tablet machines. This type of machine is widely accepted as a compaction tester to evaluate compaction properties of powders. In this type of machine, the punch is mostly driven by a simple hydraulic compression system and only the upper punch penetrates into the die to compress a powder while position of the lower punch does not change during preparation of a tablet. Single punch tablet machines can prepare tablets from a tiny amount of powder. However, the mechanism of powder densification in single punch tablet machines can be significantly different from that in rotary tablet machines due to different motion of the punches. ${ }^{5)}$ Therefore, development of a novel tablet machine which enables us to prepare tablets from a tiny amount of powders (a few grams) under the same compression mechanism as the rotary tablet machines is strongly required.

During formulation study of a tableting process, tableting troubles such as capping, binding, and sticking sometimes become a serious problem. Capping is one of the most common tableting troubles, in which cracking or lamination of a tablet is occurred. Thus, a methodology to evaluate the capping tendency has been a strong interest in pharmaceutical industries. Sugimori et al. ${ }^{6,7)}$ proposed an in-die evaluation method of capping by monitoring of a residual die wall pressure at the decompression stage. However, as pointed out by Takeuchi et al., ${ }^{8)}$ this study was conducted under the quasistatic compression/decompression conditions, and an in-die evaluation method of capping under dynamic compression/ decompression conditions have not been proposed yet.

In this paper, a novel single punch tablet machine for a tiny amount of powder was proposed. In this machine, motions of both upper and lower punches can be set arbitrarily so that a tablet can be prepared under the same compression mechanism as the rotary tablet machines, while tablets can be prepared from a tiny amount of powder. First of all, performance of the developed machine was evaluated in a continuous direct tableting test. This machine was then applied to evaluation of the capping tendency under dynamic compression/decompression conditions. A new in-die evaluation method was proposed by employing a force-displacement profile during the compression/decompression of different kinds of powder samples. 


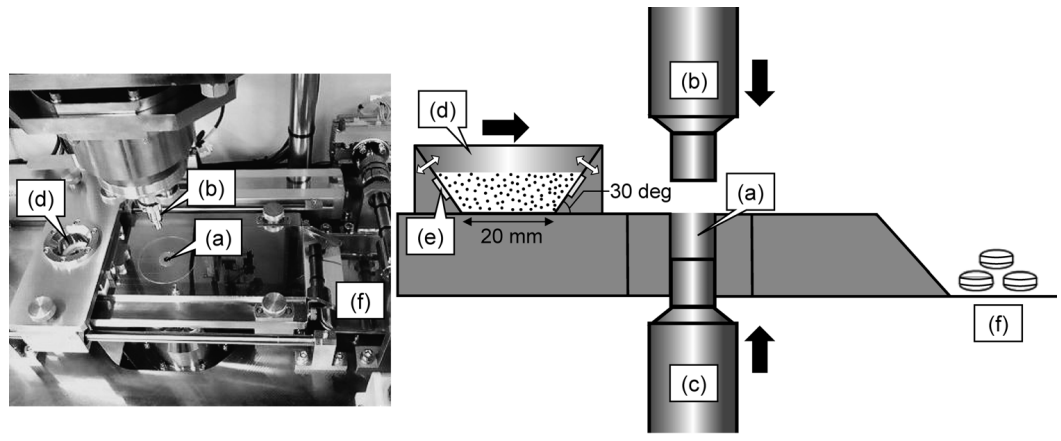

(a) die; (b) upper punch; (c) lower punch; (d) powder feeder;

(e) micro-vibrator; (f) collection table.

Fig. 1. Novel Tablet Machine for a Tiny Amount of Powder

Table 1. Formulation of Model Powder in Continuous Direct Tableting Test

\begin{tabular}{lc}
\hline \multicolumn{1}{c}{ Materials } & Mass ratio (wt\%) \\
\hline Lactose $^{a)}$ & 67.3 \\
Corn starch $^{b)}$ & 28.8 \\
Hydroxypropyl cellulose $(\mathrm{L}-\mathrm{HPC})^{c)}$ & 3.4 \\
Magnesium stearate $^{()}$ & 0.5 \\
\hline Total & 100 \\
\hline
\end{tabular}

a) 200M, DMV Pharmatose; b) Corn starch W, Nihon Shokuhin Kako Co., Ltd.; c) LH22, Shin-Etsu Chemical Co., Ltd.; d) Kishida Chemical Co., Ltd.

\section{Experimental}

Equipment Figure 1 illustrates a schematic of the developed novel tablet machine. This machine mainly consists of upper and lower punches, single die, and conical powder feeder equipped with micro-vibrators. In the die filling stage, the conical powder feeder laterally moves to cavity of the die and a powder is discharged to the die. Two electric micro-vibrators are installed at outlet of the feeder, leading to smooth powder discharge. By using this powder feeder, mass of discharged powder can be maintained constant even if a powder is poor flowability. After the die filling stage, compaction of a powder takes place. Motions of both upper and lower punches in compression and decompression stages can be set arbitrarily so that a tablet can be prepared under the same compression mechanism as the rotary tablet machines. When either maximum compression load or minimum compression thickness reaches a set-value, a compression stage is finished, followed by a decompression of a compacted powder. Finally, tablet is ejected from the die by the lower punch. The ejected tablet is conveyed by the powder feeder to a collection table. Tablets can be automatically and continuously prepared. Loads and displacements of upper and lower punches during tableting can be monitored in real-time.

The compression velocity of the developed tablet machine can be increased up to $10 \mathrm{~mm} / \mathrm{s}$. This compression velocity is so much smaller than that of conventional rotary tablet machines. Based on our preliminary investigations, however, it was found that serious capping and lamination were frequently caused in the developed tablet machine at the same high compression velocity as that of rotary tablet machines (a few hundred $\mathrm{mm} / \mathrm{s}$ ). Thus, no tablet could be successfully prepared at such high compression velocity. Consequently, we developed the tablet machine having the same motion of the punches as the rotary tablet machines, although its compression velocity was lower than that of rotary tablet machines.

Powder Samples In a continuous tableting test, a physical mixture of lactose, corn starch, and hydroxypropyl cellulose was used as a model powder. Table 1 lists formulation of the model powder. Magnesium stearate was also mixed into the model powder as a lubricant. Angle of repose of the model powder was apporoximately $60 \mathrm{deg}$, exhibiting relatively poor flowability. In experiments of capping evaluation, model pharmaceutical powders having different compaction properties were used (Table 2). Magnesium stearate was also used as a lubricant and added by an external lubrication method.

Tableting A convex-shaped tablet (6.5 mm radius of curvature) with a
Table 2. Model Powders in Capping Evaluation

\begin{tabular}{lc}
\hline \hline \multicolumn{1}{c}{ Materials } & Mean particle diameter $(\mu \mathrm{m})$ \\
\hline Lactose $^{a)}$ & 52 \\
Corn starch $^{b)}$ & 16 \\
${\text { Hydroxypropyl cellulose }(\mathrm{L}-\mathrm{HPC})^{c)}}^{c}$ & 72 \\
Microcrystalline cellulose (MCC) $^{d)}$ & 50 \\
Acetaminophen $^{e)}$ & 60 \\
\hline
\end{tabular}

a) 200M, DMV Pharmatose; b) Corn starch W, Nihon Shokuhin Kako Co., Ltd.; $c$ ) LH22, Shin-Etsu Chemical Co., Ltd.; d) Ceolus PH101, Asahikasei Chem. Ind. Co., Ltd.; e) Iwaki Seiyaku Co., Ltd.

Table 3. Operating Parameters in Continuous Direct Tableting Test

$\begin{array}{lrl}\text { Mass of a tablet (desired-value) } & 195 & (\mathrm{mg}) \\ \text { Charged mass of powder } & 10.0 & (\mathrm{~g}) \\ \text { Pre-compression load } & 3.0 & (\mathrm{kN}) \\ \text { Main-compression load } & 10.0 & (\mathrm{kN}) \\ \text { Pre-compression thickness } & 5.0 & (\mathrm{~mm}) \\ \text { Main-compression thickness } & 4.0 & (\mathrm{~mm}) \\ \text { Compression velocity } & 3.0 \quad(\mathrm{~mm} / \mathrm{s})\end{array}$

Table 4. Operating Parameters in Capping Evaluation

$\begin{array}{lcl}\text { Mass of a tablet } & 195 & (\mathrm{mg}) \\ \text { Compression load } & 5.0-15.0 & (\mathrm{kN}) \\ \text { Compression thickness } & 4.0 & (\mathrm{~mm}) \\ \text { Compression velocity } & 1.0-10.0 & (\mathrm{~mm} / \mathrm{s})\end{array}$

diameter of $8 \mathrm{~mm}$ was prepared. All tablets were prepared by a direct tableting method. In all experiments, compaction of a powder was conducted by moving of the lower punch. Table 3 lists operating parameters in a continuous direct tableting test. Desired value of mass of a tablet was set as $195 \mathrm{mg}$. Ten grams of a model powder was charged into the powder feeder. Tablets were continuously prepared by a double-compression method. After tableting, mass of each prepared tablet was measured one by one. A tablet of which the mass variation was within $\pm 2.5 \%$ of desired-value was recognized as acceptable. ${ }^{9)}$

Table 4 shows operating parameters in experiments of capping evaluation. Tablets were prepared by a single-compression method under various compression loads and punch movement velocities. A friability test ${ }^{9)}$ was conducted as an out-die evaluation of the capping tendency.

\section{Results and Discussion}

Performance of a Developed Tablet Machine in a Continuous Direct Tableting Figure 2 shows variation of mass of a tablet prepared by a developed tablet machine in a continuous direct tableting test. In this test, $195 \mathrm{mg}$ of tablets 


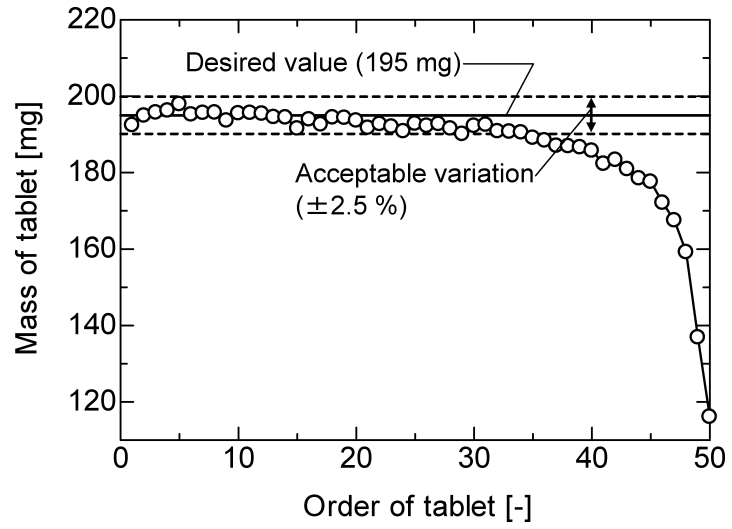

Fig. 2. Mass of Each Tablet Prepared by Continuous Direct Tableting Test

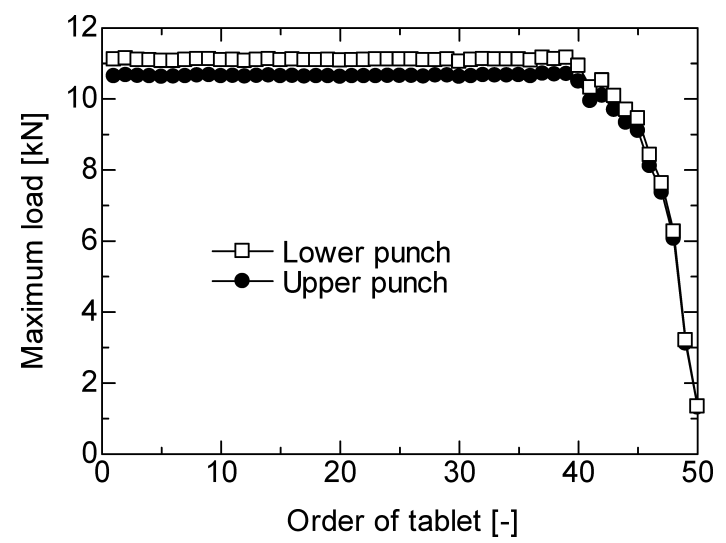

Fig. 3. Maximum Load of Main Compression in Continuous Direct Tableting Test

were prepared from $10.0 \mathrm{~g}$ of a powder. Fifty tablets can be thus ideally prepared in this test. As can be seen in Fig. 2, mass of tablets was remained within its acceptable variation range $( \pm 2.5 \%)$ until the 34 th tablet, although mass of tablets slightly decreased. This indicates that even a powder with poor flowability mass of approximately $70 \%$ of a tiny amount of $10 \mathrm{~g}(195 \mathrm{mg} \times 34)$ can be constantly discharged from the feeder. However, after the 35th tablet, mass of tablets significantly decreased and it was no longer acceptable. This is because discharge of the powder was unsteady with a decrease in weight of a powder in the feeder, resulting in insufficient filling of the die. Figure 3 indicates maximum loads of main compression acting on upper and lower punches. Maximum compression loads showed constant until the 39th tableting. This means that until the 39th tableting a powder was compressed under a constant load. Compression loads drastically decreased after 40th tableting, because a compression thickness reached its set-value due to less amount of powder filling into the die before a compression load increased to its set-value. In this case, compression of a powder was automatically stopped before a compression load reaches its setvalue. While the mass of tablet deviated from its acceptable range after 35th tableting, the compression load was kept constant even after 35th tableting and started to decrease at 40th tableting. In the developed machine, powder can be compressed at the desired compression load even if the amount of powder is less than the acceptable range, because the termination of compression stage is controlled by either

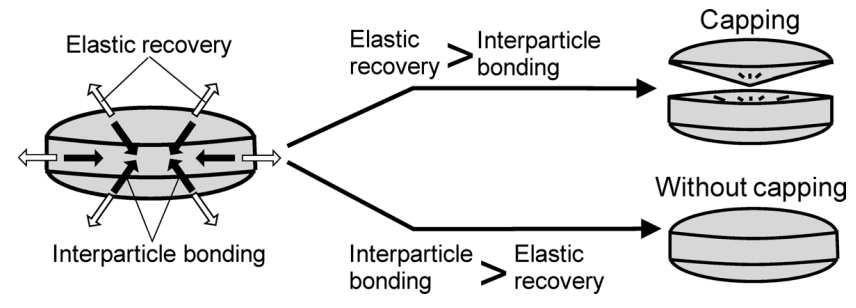

Fig. 4. Schematic of Capping Mechanism

maximum compression load or minimum compression thickness. Thus, in between 35th tableting and 39th tableting, it was considered that the minimum compression thickness was still larger than its set-value, while the amount of powder was less than the acceptable mass. This resulted in the unchanged compression load in between 35th tableting and 39th tableting.

From these results, a performance of the developed tablet machine was evaluated as follows: using this machine thirtyfour acceptable tablets $(195 \mathrm{mg} \times 34)$ can be successfully prepared in terms of mass of tablets from $10.0 \mathrm{~g}$ of a model powder with poor flowability. Accordingly, the minimum required mass of the powder to prepare one tablet having acceptable mass $(195 \mathrm{mg} \pm 2.5 \%)$ can be determined as $3.6 \mathrm{~g}$. As a verification of this minimum required mass, one tablet was actually prepared from just $3.60 \mathrm{~g}$ of the corn starch powder by the developed tablet machine. As a result, it was confirmed that mass of the prepared tablet was acceptable (191 mg). Here, corn starch powder used in this test can be categorized as a powder having poor flowability within the pharmaceutical powders. This means that most of the pharmaceutical powders have better flowability than corn starch powder. Therefore, this implies that, by using no more than $3.6 \mathrm{~g}$ of powder, most of the pharmaceutical powders can be successfully compressed into one tablet having $195 \mathrm{mg}$ by the developed tablet machine.

Evaluation of Capping Tendency As a mechanism of tablet capping, it is generally reported that the interparticle bonding of compressed powder and elastic recovery of the compacted powder during the decompression can determine the success or failure of tableting. ${ }^{10,11)}$ Figure 4 depicts a schematic of capping mechanism. When the elastic recovery is higher than the interparticle bonding the capping can be occurred, and when the interparticle bonding is higher than the elastic recovery capping cannot be occurred. In order to estimate the elastic recovery and the interparticle bonding, we here employed a force-displacement profile during the compression/decompression of a powder. Figure 5 shows a typical force-displacement profile in compression and decompression stages, monitored by the developed tablet machine. The area under the decompression curve (ADCA) is equivalent to the energy of elastic recovery $\left(E_{\mathrm{e}}\right) .{ }^{12)}$ The area of OBDO, enclosed by a compression curve of an upper punch and a decompression curve of a lower punch, is equivalent to the energy of plastic deformation of the powder bed $\left(E_{\mathrm{p}}\right){ }^{12)}$ Basically, the energy of plastic deformation can be well correlated with the energy of interparticle bonding formed by compression; the energy of plastic deformation $\left(E_{\mathrm{p}}\right)$ was thus used as a factor of the interparticle bonding.

Figures 6 and 7 show energies of plastic deformation and elastic recovery of model powders, respectively, as a function 


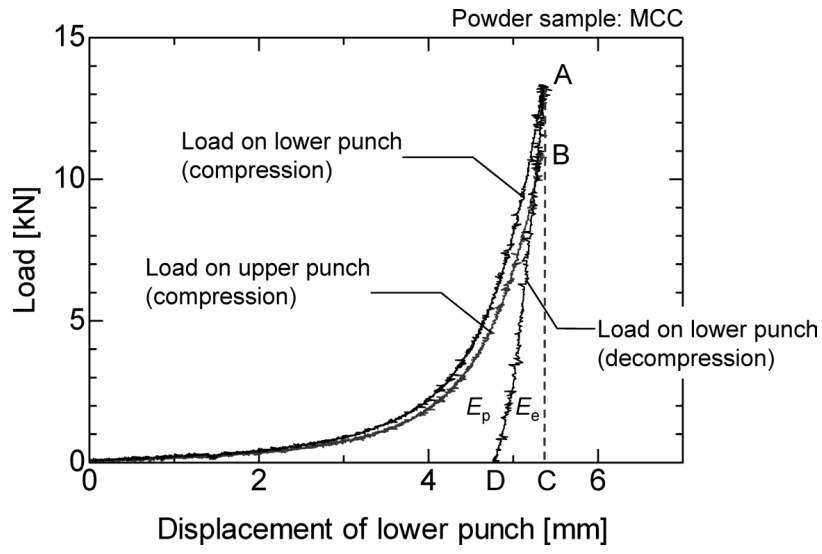

Fig. 5. Typical Force-Displacement Profile in Compression and Decompression Stages

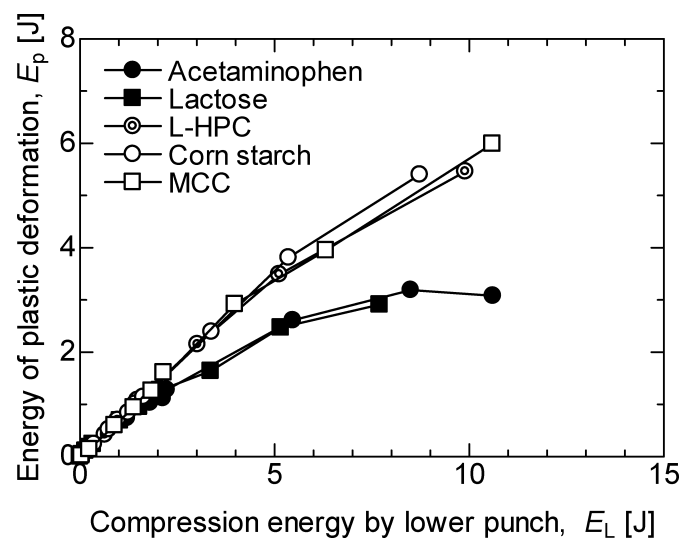

Fig. 6. Plastic Deformation Energy of Various Model Powders

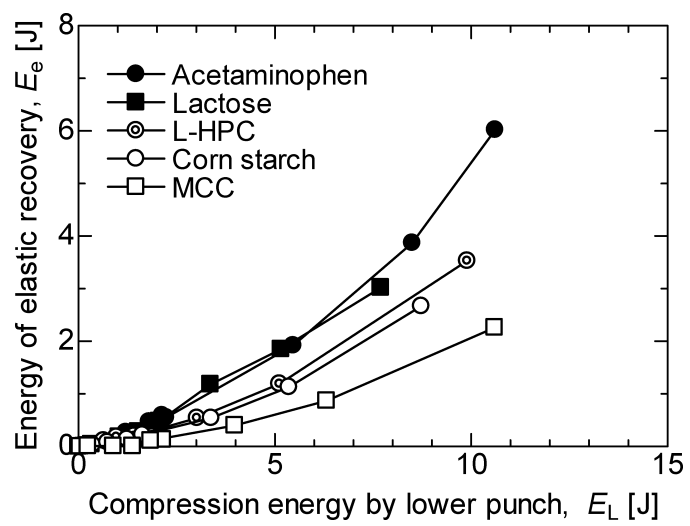

Fig. 7. Elastic Recovery Energy of Various Model Powders

of compression energy by a lower punch $\left(E_{\mathrm{L}}\right)$. The $E_{\mathrm{L}}$, which can be defined as an area under compression curve by a lower punch (OACO in Fig. 5), means total energy provided by motion of the lower punch during the compression stage. As can be seen in these results, model powders having relatively high-compactibility such as MCC exhibited higher plastic deformation energy and lower elastic recovery energy. By contrast, model powders having relatively low-compactibility such as acetaminophen showed lower plastic deformation energy and higher elastic recovery energy regardless of the compression energy by a lower punch. These results suggest that both of energies calculated from a force-

\begin{tabular}{lll}
\hline With capping & & Without capping \\
\hline - Acetaminophen & $\square$ & L-HPC \\
Lactose & $\nabla$ & Corn starch \\
& $\diamond$ & MCC \\
& $\Delta$ & Lactose \\
\hline
\end{tabular}

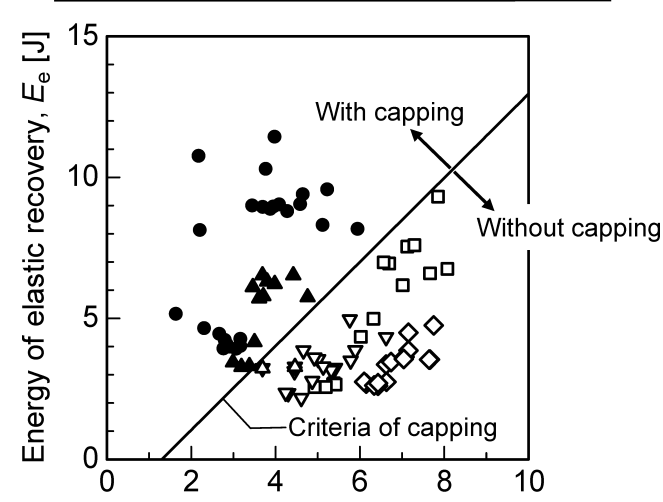

Energy of plastic deformation, $E_{\mathrm{p}}[\mathrm{J}]$

Fig. 8. Evaluation of Capping Tendency Using $E_{\mathrm{e}}-E_{\mathrm{p}}$ Diagram

displacement profile can well explain the compactibility of a powder.

To discriminate the capping tendency, a novel phase diagram consisting of energies of the elastic recovery $\left(E_{\mathrm{e}}\right)$ and the plastic deformation $\left(E_{\mathrm{p}}\right)$ was proposed. Figure 8 shows the proposed " $E_{\mathrm{e}}-E_{\mathrm{p}}$ diagram." This diagram inherently expresses a balance between $E_{\mathrm{e}}$ and $E_{\mathrm{p}}$, which are the primary factors of the capping occurrence. In Fig. 8, the closed and open plots indicate tablets with and without the capping, respectively. The tablets were prepared under the various tableting conditions as shown in Table $4 . E_{\mathrm{e}}$ and $E_{\mathrm{p}}$ were calculated in each tableting test. It should be noted that by using the $E_{\mathrm{e}}-E_{\mathrm{p}}$ diagram the capping tendency of tablets can be well discriminated regardless of tableting conditions. Tablets with capping were plotted in the upper-left region: i.e., lower $E_{\mathrm{p}}$ and higher $E_{\mathrm{e}}$ region. By contrast, tablets without capping were plotted in the lower-right region: i.e., higher $E_{\mathrm{p}}$ and lower $E_{\mathrm{e}}$ region. From this result, as shown in Fig. 8, the empirical criteria line of capping could be determined. By using the criteria line, occurrence of capping can be quantitatively discriminated by using just $E_{\mathrm{e}}$ and $E_{\mathrm{p}}$, monitored during tableting. This suggests that a new in-die evaluation is possible to quantitatively evaluate the capping tendency under the dynamic tableting conditions by using the $E_{\mathrm{e}}-E_{\mathrm{p}}$ diagram and the criteria line of capping.

\section{Conclusion}

A novel single punch tablet machine was developed. This machine can prepare tablets from a tiny amount of powder under the same compression mechanism as in the conventional rotary tablet machines. Performance of the developed tablet machine was investigated in a continuous direct tableting test. The developed tablet machine can successfully prepare thirty-four tablets $(195 \mathrm{mg} \times 34)$ using no more than $10.0 \mathrm{~g}$ of powder even if a powder has poor flowability.

We also proposed a novel in-die evaluation method of the capping tendency. A new phase diagram consisting of the energy of elastic recovery $\left(E_{\mathrm{e}}\right)$ and energy of plastic deformation $\left(E_{\mathrm{p}}\right)$, named as " $E_{\mathrm{e}}-E_{\mathrm{p}}$ diagram," was proposed. The $E_{\mathrm{e}}$ and $E_{\mathrm{p}}$ were calculated from a force-displacement profile, 
monitored by the developed tablet machine. The results indicate that using the $E_{\mathrm{e}}-E_{\mathrm{p}}$ diagram the capping tendency of tablets prepared from various powders at various tableting conditions can be well discriminated.

Acknowledgements The authors wish to acknowledge Dr. Yasuhiro Shimada (Nano-Seeds Co., Ltd., Japan) and Mr. Takaaki Seki (TokusyuKeisoku Co., Ltd., Japan) for their useful suggestions and assistances.

\section{References}

1) Anuar M. S., Briscoe B. J., Powder Technol., 195, 96-104 (2009).

2) Urabe M., Ito S., Itai S., Yuasa H., Kanaya Y., Int. J. Pharm., 263, $183-187$ (2003)

3) Ohnishi Y., Watano S., Chem. Pharm. Bull., 54, 1244-1247 (2006).

4) Tanino T., Aoki Y., Furuya Y., Sato K., Takeda T., Mizuta T., Chem. Pharm. Bull., 43, 1772-1779 (1995).
5) Palmieri G. F., Joiris E., Bonacucina G., Cespi M., Mercuri A., Int. J. Pharm., 298, 164-175 (2005).

6) Sugimori K., Mori S., Kawashima Y., Chem. Pharm. Bull., 37, 458462 (1989).

7) Sugimori K., Mori S., Kawashima Y., Powder Technol., 58, 259-264 (1989).

8) Takeuchi H., Nagira S., Yamamoto H., Kawashima Y., Int. J. Pharm., 274, 131-138 (2004).

9) Society of Japanese Pharmacopoeia, "The Japanese Pharmacopoeia 15th Eds.," The Minister of Health, Labour and Welfare, Tokyo, 2006.

10) Dwivedi S. K., Oates R. J., Mitchell A. G., J. Pharm. Pharmacol., 44, 459-466 (1992).

11) van der Voort Maarschalk K., Zuurman K., Vromans H., Bolhuis G. K., Lerk C. F., Int. J. Pharm., 140, 185-193 (1996).

12) Ragnarsson G., Sjögren J., J. Pharm. Pharmacol., 37, 145-150 (1985). 